\title{
Review on Partial Discharge Detection Techniques Related to High Voltage Power Equipment Using Different Sensors
}

\author{
MM YAACOB $^{1}$, MA ALSAEDI $^{1,2^{*}}$, JR RASHED ${ }^{2}$, AM DAKHIL $^{2}$, and SF ATYAH ${ }^{2}$ \\ ${ }^{1}$ Institute of High Voltage and High Current, Faculty of Electrical Engineering, Universiti Teknologi Malaysia \\ ${ }^{2}$ Department of Electrical Engineering, College of Engineering, Misan University, Iraq \\ *Corresponding author: MA ALSAEDI_E-mail: maliksaady@yahoo.com
}

\begin{abstract}
When operating an equipment or a power system at the high voltage, problems associated with partial discharge (PD) can be tracked down to electromagnetic emission, acoustic emission or chemical reactions such as the formation of ozone and nitrous oxide gases. The high voltage equipment and high voltage installation owners have come to terms with the need for conditions monitoring the process of PD in the equipments such as power transformers, gas insulated substations (GIS), and cable installations. This paper reviews the available PD detection methods (involving high voltage equipment) such as electrical detection, chemical detection, acoustic detection, and optical detection. Advantages and disadvantages of each method have been explored and compared. The review suggests that optical detection techniques provide many advantages in the consideration of accuracy and suitability for the applications when compared to other techniques.
\end{abstract}

Keywords: PD detection methods, power transformer, high voltage equipments

Citation: MM YAACOB, MA ALSAEDI, JR RASHED, AM DAKHIL, and SF ATYAH, "Review on Partial Discharge Detection Techniques Related to High Voltage Power Equipment Using Different Sensors," Photonic Sensors, 2014, 4(4): 325-337.

\section{Introduction}

Stability of any power system network is determined mainly by the high voltage equipment used. Failure of this equipment is normally related to partial discharge (PD) activity which deteriorates the system performance and can lead to breakdowns, fires or irreparable damage to the system. PD detection is necessary as precautionary measures to ensure that high voltage equipment insulation is not exposed to any unnecessary hazards. A good understanding of PD mechanisms, characteristics, and its development processes is essential for power system designer and power system installation maintenance engineer.

There is a high risk of insulation system dielectric instability when PD occurs. Therefore, measurement and monitoring of PD is an important preventive tool to safeguard the high-voltage equipment from wanton damage. Techniques for detecting PD depend on what different physical properties which accompany the occurrence of PD, are measured. Known physical properties which have been used in the measurement include electromagnetic emission (in the form of radio wave, light, and heat), acoustic emission (in the audible and ultra-sonic ranges), ozone formation, and the release of nitrous oxide gases [1]. The measurement level indicates the quantity and magnitude of partial discharge. Popular methods of PD detection in the high-voltage power equipment utilize electrical, chemical, acoustic or optical measurements.

Received: 27 September 2013 / Revised version: 30 May 2014

(C) The Author(s) 2014. This article is published with open access at Springerlink.com DOI: $10.1007 / \mathrm{s} 13320-014-0146-7$

Article type: Review 
Measurement of an electrical quantity is convenient and can give precise recording of PD variations in the laboratory. However, it can give an inaccurate recording for conditions at on-site (for example to monitor in-service transformers). The inaccuracy is due to the various disturbances and interferences which can result in the high environmental noise level.

Two electrical quantity measurement methods are available. They are the ultra high frequency (UHF) method and pulse capacitive coupler method. The UHF method is based on the measurement of electrical resonance at the frequency range of up to $1.5 \mathrm{GHz}$ due to PD excitation. This method is capable of detecting as well as locating a PD source $[2,3]$. The UHF method has inherent advantages such as low noise levels due to the shielding effect of the transformer and very low signal attenuation. The UHF method can also avoid local interference throughout the $100 \mathrm{MHz}$ of its operating measurement frequency band which nestles in the whole UHF band range between $300 \mathrm{MHz}$ and $1500 \mathrm{MHz}$. This method has immunity against external noise as the UHF sensor is connected inside the transformer. The connection between the UHF sensor and power transformer is non-electrical, and hence there is reliability and safety against any induced current at the power secondary.

The pulse capacitive coupler method collects and measures the PD induced current at the detection coil which has a connection loop through some impedance to the earth line [4]. Quantitative measurement has the good sensitivity and great implementation simplicity. However, it is prone to false alarm due to this high sensitivity, and it is therefore not suitable for long-term monitoring of transformers.

The chemical measurement techniques for detecting PD in the high voltage transformer are based on the collection and some chemical measurement of oil and gas samples released during the PD process. Two chemical measurement techniques are used at the present moment. They are the high performance liquid chromatography method (HPLC) and the dissolved gas analysis method (DGA). The HPLC analyses PD expelled byproducts such as degraded forms of glucose induced by degradation of insulation $[5,6]$, while the DGA analyses the accumulated volume of the gas produced by the PD. For chemical measurement techniques, sufficient expelled byproducts or gas has to be collected before analysis can begin. Hence, there is some time delay between collection and analysis. Chemical measurement techniques are therefore not suitable for real-time monitoring. Chemical as well as electrical approaches are incapable of locating the exact position of $\mathrm{PD}$ sources.

The acoustic method detects and locates the position of the PD by studying the amplitude attenuation or phase delay of the acoustic waves propagating from the PD. This mechanical wave (acoustic wave) is caused by the mechanical energy explosion due to the vaporization of the material inside the transformer tank creating a form of the pressure field [7-11]. The acoustic wave in the transformer oil can be detected using acoustic sensors (piezoelectric transducers - PZT). When the PZT is mounted outside, on the transformer wall, it will capture interferences from the very noisy environment, and this can make PZT sensors' usefulness limited. The PZT sensor can be placed inside the oil tank of the transformer to reduce the noise and attenuation of the signal [12]. The location of the PD can be estimated by measuring the time of arrival of the acoustic wave, and position information is ascertained by using sensors at multiple locations. This makes acoustic emission sensing a more preferable measuring tool in real time of PD signal detection, Measurement using the acoustic approach has an additional advantage of possessing better noise immunity for online real-time applications. Occasionally, the acoustic method experiences difficulty in locating the exact 
origin of the PD due to interference/degradation of signals from the environmental noise $[13,14]$. Here, the sensitivity is certainly compromised.

The optical method that uses the optical fiber sensor which is small in size, highly sensitive and light weight, and possesses high frequency response and significant immunity against electromagnetic interference, can measure a wide range of chemical and physical parameters at ease. Apart from PD detection and assessment [15], optical fiber acoustic sensors have been used successfully in applications such as underwater hydrophones, construction non-destructive diagnosis, material property analysis [16], traffic monitoring, and vehicle detection. Functionally, the optical detection technique is based on fiber optic intrinsic interferometers such as Michelson interferometers, Mach-Zehnder interferometers, multimode fiber and fiber optic extrinsic such as Fabry-Perot interferometric sensors. The Michelson interferometers, Mach-Zehnder interferometers sensors suffer from fringe fading problems due to random polarization rotation. The Fabry-Perot interferometric sensors are compact in size compared to the Michelson and Mach-Zehnder fibre sensors and therefore achieve virtually singlepoint measurement. Due to the measurement sensitivity, the optical method has the restricted use for PD detection

\section{Sensors for the detection of the PD phenomenon}

\subsection{Sensors for electrical quantity measurement}

PD is an electrical discharge or spark that partially bridges a small section of insulation when two conducting electrodes emerge from the separation of distinct high concentration of positive and negative charges. It is a random localized discharge formed by transient gas ionization in an insulated system when the stress voltage exceeds a certain critical value. PD often occurs when the electric field strength exceeds the breakdown strength of insulation and can lead to a flashover. The PD phenomenon causes gradual deterioration of the insulating materials, sometimes over a period of several years, leading perhaps to eventual failure.

Locating and detecting the PD in the power transformers is vital both in industries and utilities to avoid the damage of the high-voltage equipment [17, 18]. Figure 1(a) shows the UHF sensor to detect PD in gas insulated substations (GIS). This type of sensor has been proven effective not only for laboratory test but also for on-site PD. Figure 1(b) shows the UHF sensor to test the power transformer for usage on DN50/DN80 gate valve. The sensor allows alternative ways to estimate $\mathrm{PD}$, and the configuration shown is known as bushing-tap which is suited for the measurement made on galvanic connected decoupling. Figure 1(b) shows an inductive UHF sensor setup for the measurement on the power cable termination [19]. Figure 2 shows three types of UHF sensors which can be used to characterize PD on the power transformer and GIS. They are the disc-type, monopole-type, and spiral-type. The disc-type, monopole-type, and spiral-type sensors can provides the greatest energy accumulation in the laboratory test, which indicates that the sensors have the high sensitivity for detecting radiated signals [20]. Figure 3 shows a capacitive coupler for partial discharge detection in

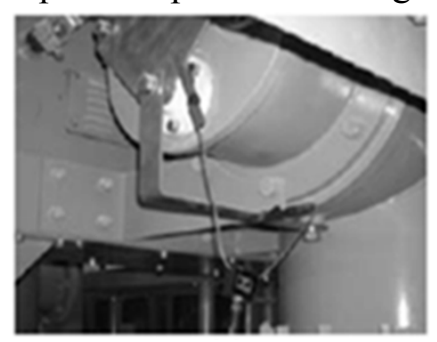

(a)

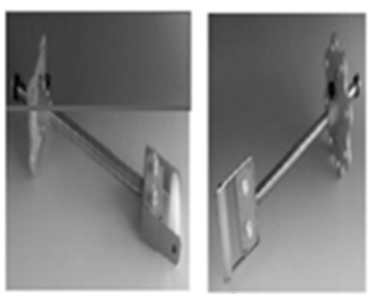

(b)

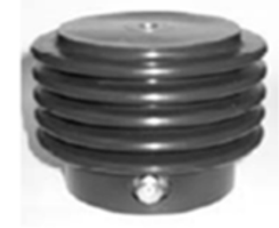

(c)
Fig. 1 Elecrical sensors: (a) UHF sensor adapted to a GIS grounding bar, (b) oil valve sensor for the power transformer reactor e.g. left side for usage on DN 50 gate valve, and right side for DN 80 gate valve, and (c) inductive UHF sensor for the power cable termination [19]. 
the high voltage cable. The $40-\mathrm{mm}$ tin tape wrapped around the exposed cable acts as a coupler for the capacitive sensor. The capacitive sensor coupler does not interfere with the measurement on the insulation of the cable because at the UHF the capacitive coupler is effectively connected and works as the power frequency ground [21].

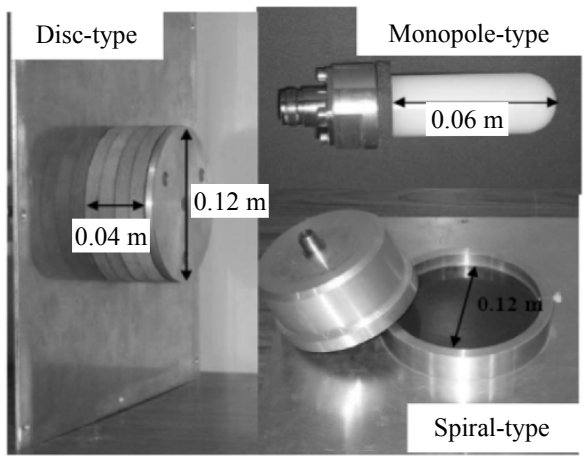

Fig. 2 Three types of UHF sensors: disc-type, monopoletype and spiral-type [20].

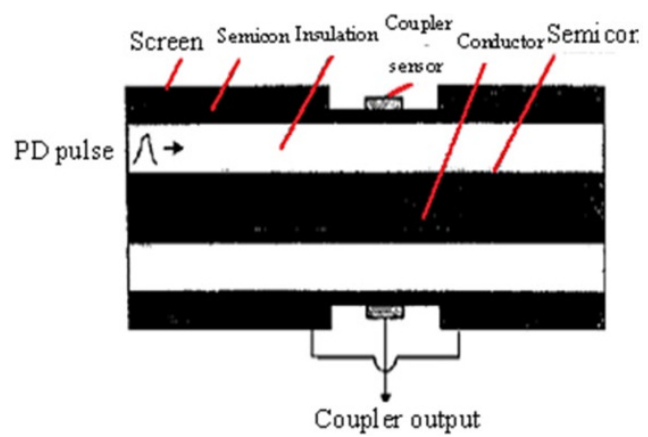

Fig. 3 Diagram of a capacitive coupler [21].

\subsection{Sensors for acoustic detection}

The acoustic technique which uses a highly sensitive piezoelectric film sensor shown in Fig. 4(a) and configured as in Fig. 4(b) has applications to the measurement of PD for the high-voltage equipment

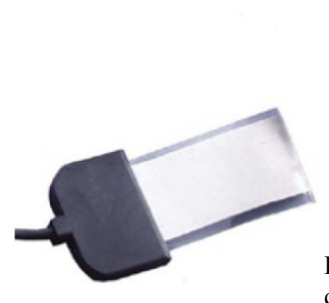

(a)

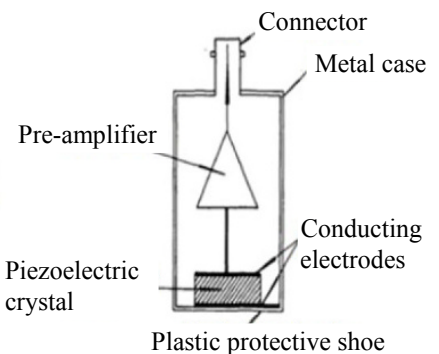

(b)
Fig. 4 Acoustic sensors: (a) piezoelectric film sensor with its connector and (b) typical design of the piezoelectric transducer sensor [22]. such as power transformers and high voltage (HV) cables. At low resonant frequencies, the piezoelectric sensor film is a crystal which is in the form of a disc, for which resonances can be calculated easily [22].

\subsection{Sensors for optical detection}

Currently, the most popular method for detecting $\mathrm{PD}$ in the high voltage transformer and other equipments such as the GIS is the optical detection method which uses the fiber intrinsic sensor coil, the detail of which is shown in Fig. 5(a). The fiber intrinsic sensor coil is made by winding $8 \mathrm{~m}$ of fiber around a former into a coil of $25 \mathrm{~mm}$ in diameter. The single mode optical fiber intrinsic sensor is based on Mach-Zehnder fiber interferometers and is normally immersed in the oil within the walls of the transformer whose PD characteristics are being measured [23]. Figure 5(b) shows the multimode optical fiber sensor for partial discharge detection in the GIS [24] while Figs. 6(a) and 6(b) show the

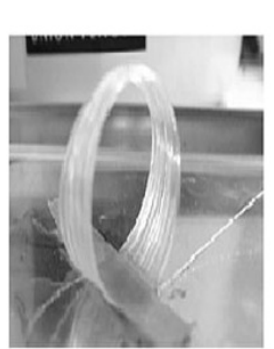

(a)

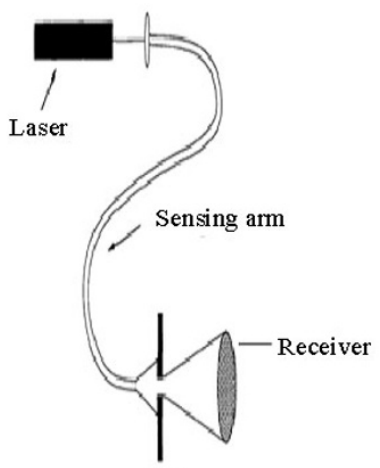

(b)
Fig. 5 Optical sensors (intrinsic): (a) optical fiber intrinsic sensor based on Mach-Zehnder fiber interferometers [23] and (b) Intrinsic multimode optical fiber sensor [24].

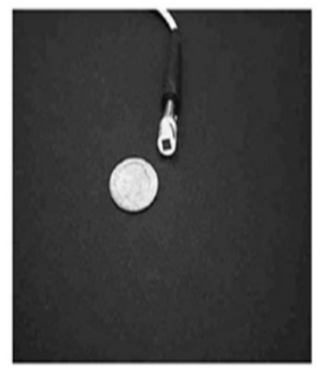

(a)

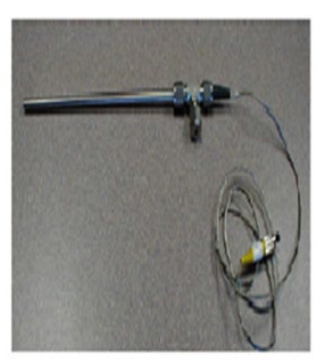

(b)
Fig. 6 Optical sensors (extrinsic): (a) extrinsic Fapry-Perot interferometer sensor [25] and (b) extrinsic microelectromechanical system sensor [25]. 
extrinsic Fapry-Perot interferometer sensor and extrinsic microelectro-mechanical system sensor which have applications to the measurement of PD of the oil cooled high voltage transformers [25].

\section{PD detection process}

\subsection{Electrical detection}

Under the influence of the intensive electric field, the PD activity starts with the transfer of electrons in short current bursts in micro void gaps. The void space charge is transferred to adjacent voids within the time frame of nanoseconds. The recharge process impulse can be detected with the aid of the $(0.1 \mathrm{nF}-1 \mathrm{nF})$ coupling capacitor. The resolution, polarity, and arrival speed of the PD induced impulse are registered [26]. With nanosecond (ns) arrival time of PD induced impulses, the signal generated by the activity of PD would be in the frequency range between $300 \mathrm{MHz}$ and $3 \mathrm{GHz}$ (UHF band). With the appropriate calibration, PD signals detected can be used as diagnosis of the insulation condition for the GIS, transformer, and cable. The detection process has the wide detection range, high sensitivity, and minimal external disturbances[27]. This property is an important consideration for monitoring insulation safety [28]. A major reason for the success in the use of the UHF method to measure PD in the GIS is the fact that the PD current pulse has short rise time $(<100 \mathrm{ps}$ in SF6) [29]. The PD signal can be detected by using the UHF sensor installed at the drain/oil valve. For the on-line measurement, disc shaped and cone shaped sensors are used but the measurement conducted in the laboratory uses cone shaped, disc shaped, and monopole sensors. The UHF method has been carried out as an electrical method to detect PD. In this method, the power supply which provides the input to the measured circuit has to have a lower discharge rate than the measured value.

\subsubsection{UHF PD detection in the power cable}

The principle of UHF PD diagnosis on the plug-in cable termination is shown in Fig. 7. The capacitive sensor (electric component) and inductive sensor (magnetic component) detect the transient electromagnetic field emitted by PD. The capacitive sensor is the copper disc with a diameter of $2 \mathrm{~cm}$. The inductive sensor consists of a two-loop winding coil. The pulses emitted by PD are pre-amplified, filtered, and processed with a computer-based oscilloscope [1]. The results of PD tests may be impaired if the readings are taken when the signal noise ratio (SNR) is poor, that is when the measurement frequency is less than $500 \mathrm{kHz}$. The proper measurement is carried out at the higher SNR when the measurement frequency is increased more than $500 \mathrm{kHz}$ and by selective tuning to the ultra wide band signal. UHF PD diagnosis works well because the attenuation of PD pulses along a power cable is high in the high frequency spectrum. The UHF technique is a good method to check on accessories of the power cable such as terminations and joints.

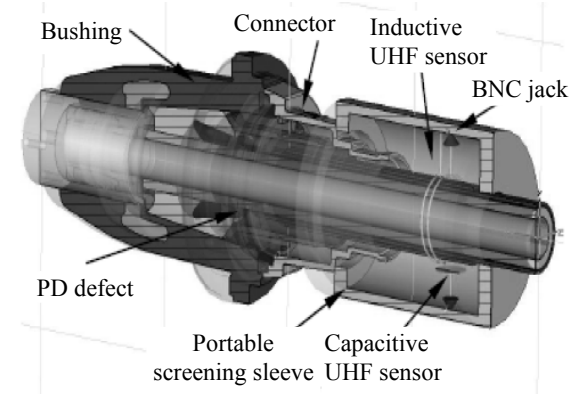

Fig. 7 Plug-in cable termination and principle of the UHF PD diagnostics [1].

Figure 8 is a schematic diagram of the UHF PD measurement system for the power cable. The system consists of a UHF PD sensor, a pre-amplifier, and a PD measurement outfit. The UHF PD sensor is connected to the GIS-cable termination, and the output of this sensor is connected to a high-pass filter via the coaxial cable to limit the operating frequency range within $300 \mathrm{MHz}$. It is also connected to a pre-amplifier to increase the SNR. The output of the pre-amplifier is then connected to the PD measurement outfit in which the main 
element is a system which does PD pulse processing [30].

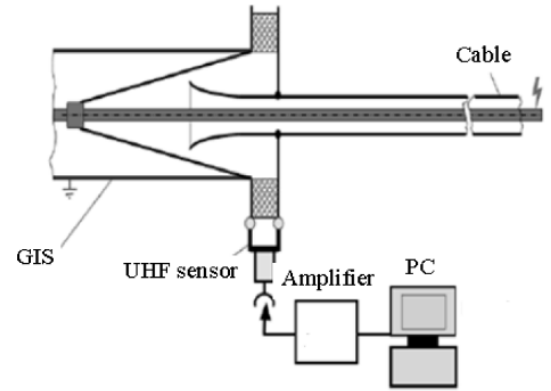

Fig. 8 UHF PD sensor connected at the high voltage-andextra high voltage-termination [30].

\subsubsection{UHF PD detection in GIS}

The PD pulse current has a short rise time, less than a nanosecond (ns) in SF6 which is used as an insulating gas. The electromagnetic wave generated by PD has a frequency component in the UHF band. The electromagnetic wave (UHF signals) emitted by PD propagates over a long distance in the GIS tank without much attenuation and can be detected and captured by the antenna and sensor. These UHF signals will not be interfered by any extraneous broadcast wave or telecommunication signal if their frequencies are sufficiently high. For the GIS, the risk assessment of breakdown is easy to determine due to information on PD phenomena in SF6 gas [31]. The accuracy of PD localization can be improved, and the maintenance efficiency in the GIS can be enhanced if the UHF PD measurement method is combined with the acoustic measurement technique. The metallic enclosure can cause power dissipation resulting in significant attenuation of the PD electromagnetic wave. Experiments have shown that very low attenuation coefficient of $2 \mathrm{~dB} / \mathrm{km}$ is normally present (theoretical attenuation coefficient value of about $1 \mathrm{~dB} / \mathrm{km}-2 \mathrm{~dB} / \mathrm{km}$ has been quoted) [27]. Figure 9 shows a typical measurement setup for UHF PD detection in the GIS. The system consists of a UHF field probe sensor, a pre-amplifier, a digitizer, and a personal computer. The signal coming from the sensor is input to a pre-amplifier which has the $25-\mathrm{dB}$ gain and a bandwidth of $1 \mathrm{GHz}$.
In the test, the digitizer captures the UHF signal using a trigger delay sampling of $20 \mathrm{~ns}$ in each step [32].

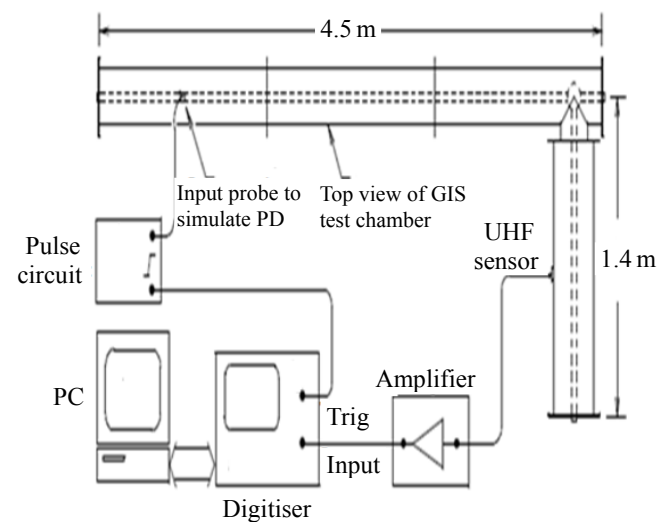

Fig. 9 Measurement equipment and a typical GIS test chamber [32].

\subsubsection{UHF PD detection in the transformer}

The UHF PD detection in the high voltage transformer is a routine maintenance and monitoring procedure. For the UHF PD detection technique of transformers, the measurement process experiences the low noise level due to shielding. The signal attenuation of oil insulation is low, and the measurement sensitivity at the on-site condition is good. The laboratory setup for the PD measurement in transformers consists of a half closed metallic tank with dimensions $(1.0 \mathrm{~m} \times 0.5 \mathrm{~m} \times 0.5 \mathrm{~m})$, a needle sphere PD source, and two similar disc-sensors. The bandwidth of the transient reading record is normally $3 \mathrm{GHz}$, and no additional amplification is required as the quality of the output from the pre-amplifier is good. The UHF technique is increasingly used to test power transformers and has the sensitivity better than AE [33]. Figure 10 is a typical UHF monitoring system setup. The signals from the sensors are filtered and amplified, then detected and digitized. The main reason for digitization is to allow the signal to be used dynamically. The phase reference and clock provide additional information that is logged in with the digitized data. The recorded pulse of the partial discharge can be considered as coming from a point source in real time. The amplitude of this pulse has 
been calibrated against the energy of the UHF signals [2]. The coupling capacitor allows UHF signals to pass through to the pre-amplifier and at the same time blocks all low frequency interferences (please refer to Fig. 11). The test rig is corona-free and can accommodate a range of transformer sizes with sufficient interconnections.

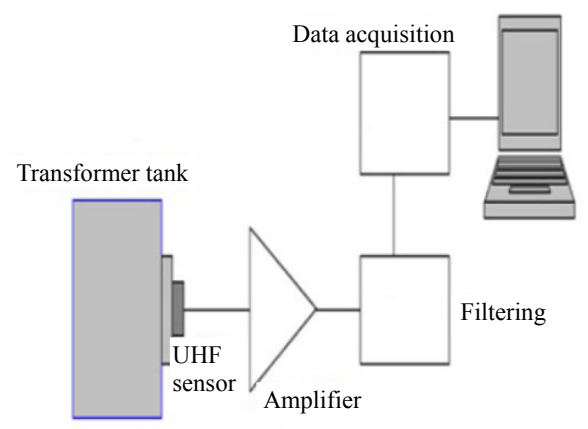

Fig. 10 Principle of a typical UHF of the PD monitoring system [2].

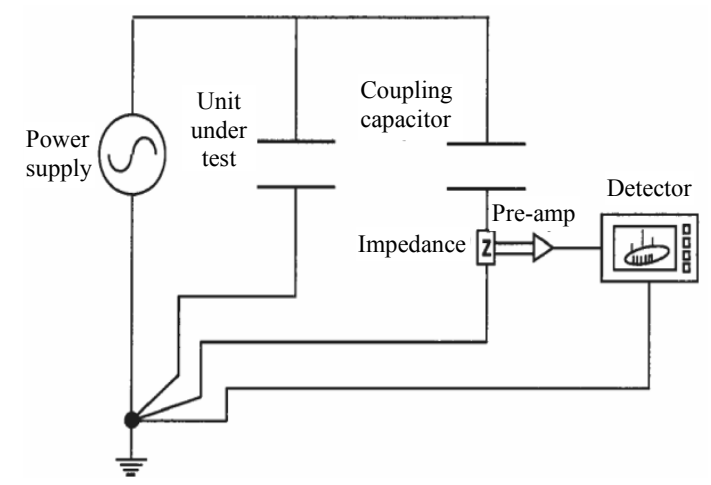

Fig. 11 Principle of a typical UHF of [2].

\subsection{Chemical detection}

The PD signal can also be detected chemically by observing the chemical changes in the composition of insulating materials in the transformer and in the GIS. Chemical detection employs high performance liquid chromatography (HPLC) or dissolve gas analysis (DGA). In the DGA test, oil from the tank of an oil-cooled transformer or an insulation material from the GIS is sampled and analyzed to quantify the different levels of gases such as methane, acetylenes, carbon dioxide, ethylene, and hydrogen [34]. These levels should not exceed those which have been specified for the condition when oil insulation or an insulation material breaks down. Unfortunately, the readings from DGA, the levels of dissolved gases, and the types of fault have not been scientifically and experimentally correlated and calibrated. The HPLC test measures the byproducts of insulation breakdown of the transformer or GIS. The insulation breakdown of the transformer produces glucose. Collection of sufficient quantity of byproducts of insulation breakdown and analysis takes too long for real-time monitoring. Both DGA and HPLC tests suffer from the same uncertainty problem as there is no known standard correlation between the amount of glucose discharged during insulation breakdown and the type and level of severity of the high voltage transformer fault. The chemical method is unable to locate the position of the PD signal source or the extent of insulation damage with some degree of accuracy. The chemical method cannot be used for real-time and online monitoring purposes.

\section{Chemical detection of PD in transformers}

Figure12(a) shows a hydrogen-oil detector. The semi-permeable membrane is placed in the oil tank of the transformer and then connected to a portable gas chromatograph. The portable gas chromatograph allows the measurement of the concentration of the hydrogen gas at a regular interval of a few hours. The second commercial model of the DGA test for transformers which use fuel-cell type detectors [30] are shown in Fig. 12(b).

\subsection{Acoustic detection}

The frequency of signal emission from PD in the oil is in the wideband range $(10 \mathrm{kHz}-500 \mathrm{kHz})$ which can be detected by the PZT ultrasound sensor. Normally, for many detection and monitoring of PD, acoustic sensors are mounted outside the high-voltage equipment. Acoustic detection which uses electromagnetic interference for its measurement requires the installation of a PD coupler. Mechanical energy explosion due to the vaporization of the material in the oil creates a 
pressure field forming the acoustic wave. The capacitive nature of PD is unimportant because the sensitivity of the acoustic method does not vary with the test object capacitance [31]. The PD acoustic signal detection method has the lower sensitivity when compared to the PD electrical signal detection method. However, the location where partial discharge arises can be estimated by measuring the differences of acoustic signal time of arrival (TOA) at more than three multiple sensors deployed.

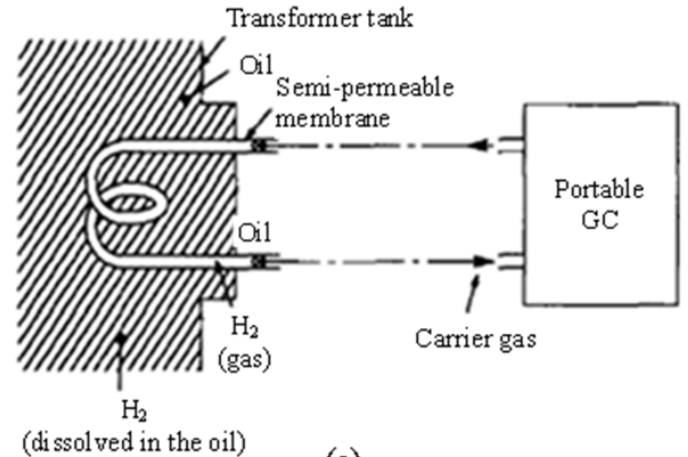

(a)

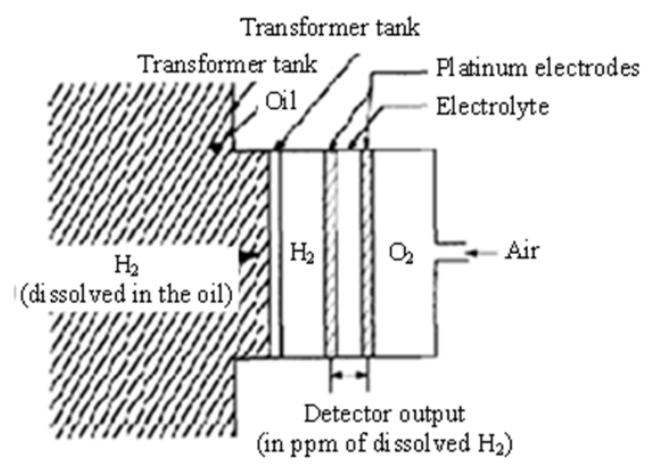

(b)

Fig. 12 Chemical technique of PD detection: (a) hydrogenoil-detector and (b) hydrogen-in-oil detector continuous monitoring.

\subsubsection{Acoustic technique for the detection of PD in the GIS}

The GIS has been constructed as a closed structure filled with the pressurized gas. The gas SF6 has a better insulation property as compared to air. The GIS system is compact physically, reliable in the function of all its contact points, and safe to use. PD in the GIS can be detected by measuring its acoustic emission (AE) picked up from the walls of the GIS metal enclosure. The acoustic waves emitted by PD are radically symmetric and have a broadband range of $20 \mathrm{kHz}-250 \mathrm{kHz}$. The GIS practical implementation with the contact sensor is illustrated in Fig. 13.

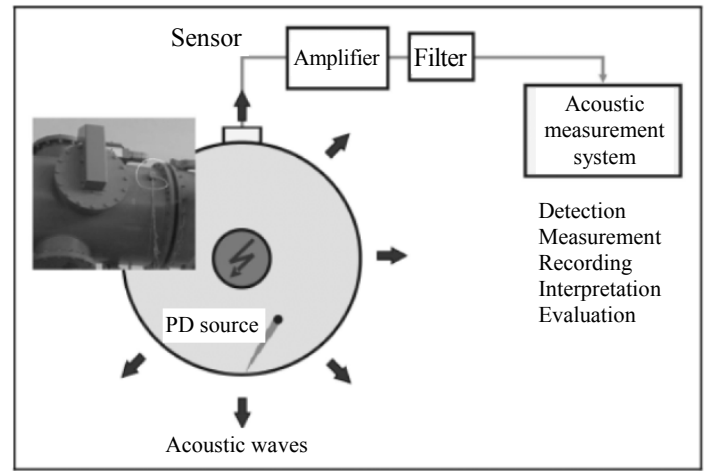

Fig. 13 GIS practical implementation with the contact sensor.

\subsubsection{Acoustic technique in the detection of PD for an oil cooled transformer}

The life of a transformer has a considerable economic impact on the operation of an electrical power network. The most important parameter which determines a transformer's life expectancy is the maximum value of temperature, and its insulation can be tolerated during operation. Any failure reduces network reliability and increases maintenance costs. For an oil cooled transformer, the PD generated acoustic signal is detected by a de-coupler and enhanced with the help of a low-noise amplifier to ensure that the sensitivity of the reading is high before it is analyzed. In the laboratory tests, PD has to be simulated. For the laboratory simulation, three types of electrode systems are employed. They are the plane-plane, needle-plane, and wire-wire structures which when excited will produce the simulated partial discharge in the oil within the transformer walls. When the alternating current $(\mathrm{AC})$ voltage is increased from 0 to $50 \mathrm{kV} \mathrm{rms} \mathrm{(please} \mathrm{refer} \mathrm{to} \mathrm{Fig.} \mathrm{14),} \mathrm{PD} \mathrm{is}$ generated at the electrode system which is immersed in the oil. An acoustic emission sensor is installed on the outer surface of the tank. This sensor will detect the PD induced acoustic signals and transmit them to an oscilloscope through a low-noise amplifier 
which has a 3-dB mid-band frequency bandwidth of $1.6 \mathrm{kHz}$ to $1.6 \mathrm{MHz}$. The frequency ranges of the acoustic signals are $45 \mathrm{kHz}$ to $25 \mathrm{kHz}$ for a plane-plane electrode system, $60 \mathrm{kHz}-279 \mathrm{kHz}$ for the needle-plane electrode system, and $50 \mathrm{kHz}-$ $180 \mathrm{kHz}$ for the wire-wire electrode system. The frequencies where detected at $145 \mathrm{kHz}$ for the needle-plane electrode and $121 \mathrm{kHz}$ for the wire-wire electrode. The location of PD can be determined within $1 \%$ error if three acoustic sensors are used to pick up PD induced acoustic signals [32].

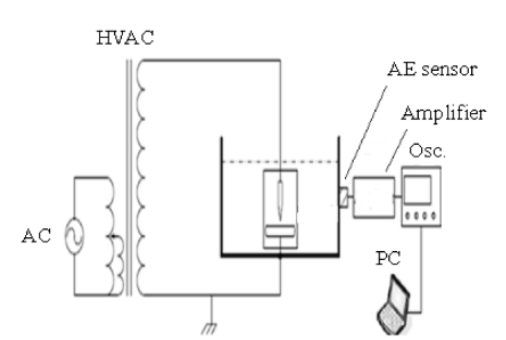

Fig. 14 Configuration of the experimental apparatus.

\subsubsection{Acoustic technique to detect PD in the HV cable}

The acoustic wave which is propagated through the cable material, interface and the high voltage cable joint may be attenuated and may result in the low detection sensitivity. Care must be taken to ensure that the attenuation do not degrade the wave too much in order that on-line measurement can quantify the PD. The acoustic technique do not suffer from any significant electrical interference. This is a real advantage especially when the PD measurements are carried out on site. The location of PD is ascertained by analyzing the time of flight between the pulse of electrical discharge and the signals collected by the different acoustic emission sensors. Figure 15 shows the position arrangement of the acoustic sensor on to the high voltage cable joint. The piezoelectric sensor collects the acoustic signal in the cable joint from the mechanical pressure it detects. The silicon grease which is spread between the piezoelectric sensor and the cable joint reduces the air gap which can cause the attenuation of acoustic signals.

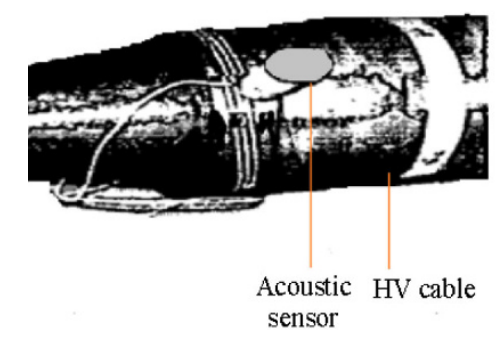

Fig. 15 Acoustic sensor was bounded with cable joint.

\subsection{Optical detection}

The optical fiber has been used as a sensor for a long time because of its many advantages such as safety from electrical spark, resistance to chemical corrosion, response capability to a wide range of measurements, ability to stand high temperature, small size, large bandwidth, and high sensitivity. Optical detection is based on the fractional changes in optical parameters such as the wavelength, intensity, polarization, and phase. Hence, it is possible to get four types of optical sensors; namely spectrum based sensor, intensity based sensor, polarization based sensor, and interferometric based sensor. The fiber optic acoustic sensor includes the optical fiber intrinsic sensor such as Michelson interferometers, Mach-Zehnder interferometers, multimode fiber and fiber optic extrinsic such as Fabry-Perot interferometric sensors. The fiber optic acoustic sensor combines the acoustic and optical methods. The detection process of this method is based on the photo elastic effect of the silica fiber. The acoustic wave that is incident on the optical fiber will cause distortion of the optical fiber structure. This distortion will change the fiber length and fiber refractive index. This change can create a modulation effect on a laser beam which passes through the fiber. Due to the low photo elastic effect of the silica fiber, the sensitivity needs to be increased. 


\subsubsection{Optical detection of PD in the transformer using Mach-Zehnder iinterferometers}

The location of PD in a large oil-filled power transformer makes it very difficult to use an external acoustic sensor. It will then be necessary to use a fiber-optic coil as an acoustic sensor to locate and detect the PD with the adequate sensitivity. An earlier method of acoustic detection is based on fiber optic essential interferometers such as the fiber Michelson and Mach-Zehnder interferometers. This essential fiber sensor uses a single mode fiber and laser. From the source, the light is split into two fibers with equal intensity of $3 \mathrm{~dB}$ via a fiber coupler. The first fiber is referred to as the reference arm, while the second fiber is referred to as the sensing arm. To generate interference signals, the lights in the two arms are recombined either by transmission such as the Mach-Zehnder interferometer or by reflection such as the Michelson interferometer. The reference arm has the original light source while the sensing arm is allowed to be interfered by the acoustic wave induced by the PD. From the sensing arm, the original light source will have been modulated by the acoustic wave. When a long fiber is used during sensing, the essential fiber interferometer sensor has a very high sensitivity. Figure 16 shows an experimental setup for PD signal detection using the optical fiber sensor coil in the transformer oil. The system consists of a coherent light source $\mathrm{He}-\mathrm{Ne}$ laser $(633 \mathrm{~nm})$, a single mode optical fiber, an optoelectronic transducer to convert the optical signal to the electrical signal, a high voltage source and two electrodes to generate the simulated PD which will produce the acoustic emission. When the acoustic wave impinges on the fiber optic sensor coil, the phase of the optical signal in the sensor coil will change. The optical signal in the sensor coil will be modified in a modulating fashion due to successive phase changes. The light source from the sensor coil will then be converted to the electrical signal which is then amplified before being observed (eg. using a digital oscilloscope) [33].

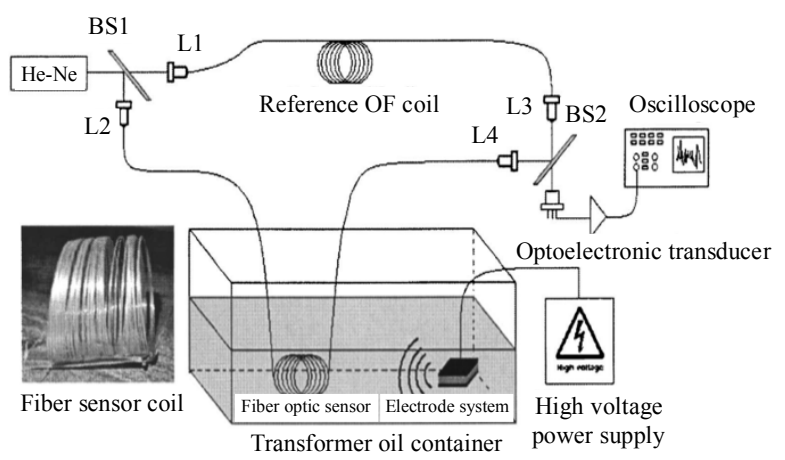

Fig. 16 Experimental setup for detection of PD generated in laboratory condition using the Mach-Zehnder interferometer.

\subsubsection{Optical detection of PD in transformer using Fabry-Perot interferometric sensor}

The Fabry-Perot interferometric sensor has been used in many applications, one of which is the detection of the PD induced acoustic wave [34]. The sensing element (small in size) known as the Fabry-Perot cavity is composed using two reflecting surfaces. Figure 17 shows the basic principle of the Fabry-Perot interferometer sensor. The system consists of a light source launched into a $2 \times 2$ coupler. One output of the $2 \times 2$ coupler goes to a single-mode fiber which is connected to the sensor head while the other arm leads to a photo detector where the optical signal is converted to the electrical signal. The electrical signal is sent to an amplifier and subsequently to the input of a digital

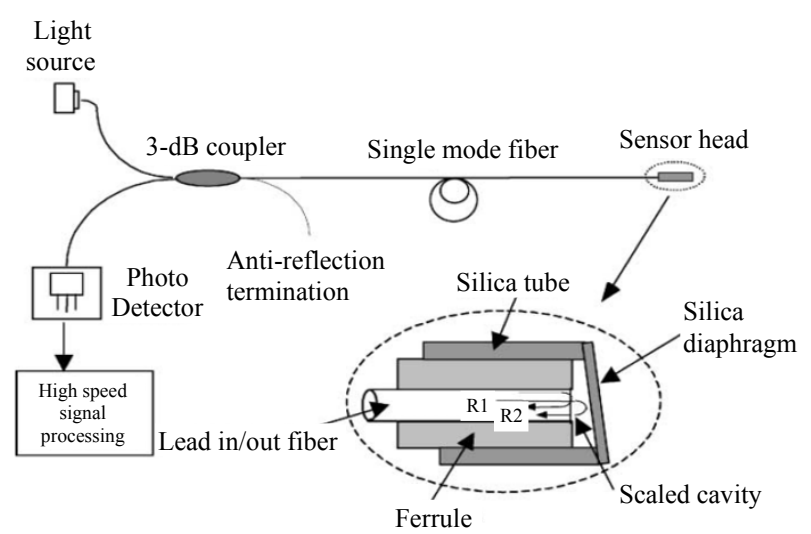

Fig. 17 Illustration of principle of the Fabry-Perot interferometric sensor. 
oscilloscope or a high speed signal processor. The sensor lead uses a silica diaphragm and single-mode fiber to form the Fabry-Perot interferometer sensor. It has been proven from several tests that the Fabry-Perot interferometer sensor is an excellent candidate to detect the acoustic signal induced by PD in transformers. A comprehensive summary of

Table 1 Overview of PD detection methods.
PD detection methods are displayed in Table 1. The comparison exercise has shown that optical detection techniques for PD detection are better than non-optical ones. The most overriding advantage is, of course, that they are safe from any unexpected electrical spark and that they are resistant to chemical corrosion attacks.

\begin{tabular}{|c|c|c|c|c|}
\hline Method & Advantage & Disadvantage & Sensor & Application \\
\hline \multirow[t]{7}{*}{ Electrical } & $\begin{array}{l}\text { Good recording of PD signals in the } \\
\text { laboratory environment }\end{array}$ & Very difficult to apply on site & $\begin{array}{l}\text { Coupling } \\
\text { Capacitance }\end{array}$ & Cable \\
\hline & High sensitivity & False alarm due to high sensitivity & UHF adapted & GIS \\
\hline & $\begin{array}{l}\text { Low noise levels due to shielding effect of } \\
\text { the transformer }\end{array}$ & $\begin{array}{l}\text { Not suitable for long-term monitoring of } \\
\text { transformers }\end{array}$ & Directional coupler & Cable \\
\hline & Very low signal attenuation & Influenced by electromagnetic interference & DN50,80 & $\begin{array}{l}\text { Transformer } \\
\text { Cable }\end{array}$ \\
\hline & High precision measurements & Vulnerable to noise & $\begin{array}{l}\text { Inductive UHF } \\
\text { Disc-type }\end{array}$ & Transformer \\
\hline & & & Monopole-type & Transformer \\
\hline & & & Spiral-type & Transformer \\
\hline \multirow[t]{2}{*}{ Chemical } & $\begin{array}{l}\text { Good recording of PD signals in the } \\
\text { laboratory environment }\end{array}$ & $\begin{array}{l}\text { Level of dissolved gas has no correlation with } \\
\text { the specific type of fault }\end{array}$ & Chemical sample & Transformer \\
\hline & High sensitivity & $\begin{array}{l}\text { Creates uncertainty because there is no known } \\
\text { relationship between the level of glucose and } \\
\text { the severity of insulation breakdown }\end{array}$ & & \\
\hline \multirow[t]{3}{*}{ Acoustic } & Good results in real time & Low sensitivity & PZT & Transformer \\
\hline & $\begin{array}{l}\text { Has noise immunity for application to do } \\
\text { online partial discharge detection }\end{array}$ & $\begin{array}{l}\text { Interference of signals by environmental } \\
\text { noise }\end{array}$ & & \\
\hline & $\begin{array}{l}\text { Position information of } \mathrm{PD} \text { is possible } \\
\text { using sensors at multiple locations }\end{array}$ & & & $\begin{array}{l}\text { GIS } \\
\text { Cable }\end{array}$ \\
\hline \multirow[t]{6}{*}{ Optical } & $\begin{array}{l}\text { A wide range of chemical and physical } \\
\text { parameters can be used for the detection } \\
\text { process }\end{array}$ & $\begin{array}{l}\text { No disadvantage has been mentioned in } \\
\text { current literature }\end{array}$ & $\begin{array}{l}\text { Mach-Zehnder fiber interferometers } \\
\text { Multimode fiber }\end{array}$ & Transformer \\
\hline & Small size & & Fabry-Perot interferometers & $\begin{array}{l}\text { GIS } \\
\text { Transformer }\end{array}$ \\
\hline & High sensitivity & & & \\
\hline & Light weight & & & \\
\hline & High frequency response & & & \\
\hline & Immune to electromagnetic interference & & & \\
\hline
\end{tabular}

\section{Conclusions}

The problems associated with PD for the high voltage equipment and high voltage power systems have not been ignored and will never be ignored by high voltage system designers and high voltage system maintenance engineers. When it comes to high voltage, PD identification and PD monitoring make economic sense low and many years to come. How reliable, how quick PD can be measured and what sensors to use have become hot issues. This paper has gone to some lengths to present the many different ways by which PD can be measured. With the technical progress, it is now possible to put 
suitable sensors directly into the H. V. electrical equipment. The online PD monitoring system for the H.V. electrical equipment can be used to detect occurrences before breakdown happens. At the present state of the technology, it does appear that optical detection techniques have more advantages then non-optical ones. The optical detection and measurement method for PD may be considered as the future technique which will give a more precise prediction of preventive measures which can avoid time and money losses when operating the H. V. power equipment and systems.

\section{Acknowledgment}

The author gratefully acknowledges the Faculty of Electrical Engineering, Universiti Teknologi Malaysia for giving the support in this study.

Open Access This article is distributed under the terms of the Creative Commons Attribution License which permits any use, distribution, and reproduction in any medium, provided the original author(s) and source are credited.

\section{References}

[1] S. Tenbohlen, D. Denissov, S. M. Hoek, and Z. Ring, "Partial discharge measurement in the ultra high frequency (UHF) range," IEEE Transactions on Dielectrics and Electrical Insulation, 2008, 15(6): 1544-1552.

[2] M. D. Judd, L. Yang, and I. B. B. Hunter, "Partial discharge monitoring for power transformers using UHF sensors part 1: sensors and signal interpretation," IEEE Electrical Insulation Magazine, 2005, 21(2): 5-14.

[3] M. D. Judd, L. Yang, and I. B. B. Hunter, "Partial discharge monitoring for power transformers using UHF sensors part 2: field experience," IEEE Electrical Insulation Magazine, 2005, 21(3): 5-13.

[4] J. E. Timperley, A. Electric, and P. Service, "Power apparatus and systems," IEEE Transactions on Power Apparatus and System, 1983, PAS-102(3): 693-698.

[5] R. Bartnikas, "Partial discharges their mechanism, detection and measurement," IEEE Transactions on Dielectrics and Electrical Insulation, 2002, 9(5): 763-808.

[6] S. Karmakar, N. K. Roy, and P. Kumbhakar, "Partial discharge measurement of transformer with ICT facilities," in International Conference on Power
Systems 2009, Kharagpur, India, Dec. 27-29, pp. 1-5, 2009.

[7] R. T. Harrold, "Acoustical technology applications in electrical insulation and dielectrics," IEEE Transactions on Electrical Insulation, 1985, EI-20(1): 3-19.

[8] L. E. Lundgaard, "Partial discharge. XIII. acoustic partial discharge detection-fundamental considerations," IEEE Electrical Insulation Magazine, 1992, 8(4): 25-31

[9] B. R. Varlow, D. W. Auckland, C. D. Smith, and J. Zhao, "Acoustic emission analysis of high voltage insulation," IEE Proceedings Science, Measurement and Technology, 1999, 146(5): 260-263.

[10] R. T. Harrold, "Acoustic waveguides for sensing and locating electrical discharges in high voltage power transformers and other apparatus," IEEE Transactions on Power Apparatus and Systems, 1979, PAS-98(2): 449-457.

[11] M. Leijon, L. Ming, and T. Bengtsson, "PD source identification in solids," in IEEE International Symposium on Electrical Insulation, Conference Record of the 1992, Baltimore, USA, Jun. 7-10, pp. 415-418, 1992.

[12] X. Wang, B. Li, Z. Liu, H. T. Roman, O. L. Russo, K. $\mathrm{K}$. Chin, et al., "Analysis of partial discharge signal using the Hilbert-Huang transform," IEEE Transactions on Power Delivery, 2006, 21(3): 1063-1067.

[13] E. T. N. E. Howells, "Location of partial discharge sites in on-line transformers," IEEE Transactions on Power Apparatus and Systems, 1981, PAS-100(1): 158-162.

[14] P. M. Eleftherion, "Partial discharge XXI: acoustc emisson-based PD source location in transformer," IEEE Electrical Insulation Magazine, 1995, 11(6): 22-26.

[15] H. Search, C. Journals, A. Contact, M. Iopscience, S. Mater, and I. P. Address, "Optical fiber sensing technique for impact detection and location in composites and metal specimens," Smart Materials and Structures, 1995, 4: 93.

[16] N. Furstenau, M. Schmidt, H. Horack, W. Goetze, and W. Schmidt, "Extrinsic Fabry-Perot interferometer vibration and acoustic sensor systems for airport ground traffic monitoring," IEE Proc -Optoulectron, 1997, 144(4): 134-144.

[17] S. M. Markalous, Z. Ring, S. Tenbohlen, and K. Feser, "Detection and location of partial discharges in power transformers using acoustic and electromagnetic signals," IEEE Transactions on Dielectrics and Electrical Insulation, Dec. 22, pp. 1576-1583, 2008.

[18] T. Pinpart and M. D. Judd, "Experimental comparison of UHF sensor types for PD location applications," in IEEE Electrical Insulation Conference 2009, Montreal, Canada, May. 31-Jun. 3, 
pp. 26-30, 2009.

[19] A. E. D. Y. Tian and P. L. Lewin, "Comparison of on-line partial discharge detection methods for HV cable joints." IEEE Transaction on Dielectric an Electric Insulation, 2002, 9(4): 604-615.

[20] R. Dukes, B. Sc, D. Ph, E. A. Culpan, and C. Eng, "Acoustic emission: its techniques and applications," Physical Science, Measurement and Instrumentation, Management and Education Reviews, IEE Proceedings A, 1984, 131(4): 241-251.

[21] H. L. Rivera, C. Maci-Sanahuja, and J. A. Garc-Souto, "Detection and wavelet analysis of partial discharges using an optical fibre interferometric sensor for high-power transformers," Journal of Optics A Pure and Applied Optics, 2003, 5(1): 66-72.

[22] J. A. Cosgrave, A. Vourdas, G. R. Jones, J. W. Spencer, M. M. Murphy, and A. Wilson, "Acoustic monitoring of partial discharges in gas insulated substations using optical sensors," IEE Proceedings A Science, Measurement and Technology, 1993, 140(5): 369-374.

[23] A. Wang, Y. Liu, J. Deng, H. Xiao, W. Huo, M. Luo, et al., "Optical fiber sensor-based detection of partial discharges in power transformers," Optical Fibers and Applications, 2001, 33(5): 305-311.

[24] T. Huecker, "Application of uhf partial discharge monitoring and expert system diagnosis," in Conference Record of the 1998 IEEE International Symposium on Electrical Insulation, Arlington, USA, Jun. 7-10, pp. 61-64, 1998.

[25] M. D. Judd, "High bandwidth measurement of partial discharge current pulses," in Conference Record of the 1998 IEEE International Symposium on Electrical Insulation, Arlington, USA, Jun. 7-10, pp. 436-439, 1998.

[26] M. Boltze, S. M. Markalous, A. Bolliger, O. Ciprietti, J. Chiu, L. Gmbh, et al., "On-line partial discharge monitoring and diagnosis at power cables," in 76th
Annual Proceeding of Electrical Insulation, 2009.

[27] R. Sarathi, A. V. Giridhar, and K. Sethupathi, "Analysis of partial discharge activity by a conducting particle in liquid nitrogen under $\mathrm{AC}$ voltages adopting UHF technique," Cryogenics, 2010, 50(1): 43-49.

[28] Z. B. Shen and S. Member, "Localization of partial discharges using UHF sensors in power transformers," in Conference on Power Engineering Society General Meeting, 2006, IEEE, Montreal, Canada, pp. 1-6, 2006.

[29] I. J. J. Kemp, "Partial discharge plant-monitoring technology: present and future developments," IEE Proceedings Science, Measurement and Technology, 1995, 142(1): 4-10.

[30] G. Belanger and M. Duval, "Monitor for hydrogen dissolved in transformer oil," comfer IEEE Transactions on Electrical Insulation, 1977, 69(5): 334-340.

[31] G. S. Kil, I. K. Kim, D. W. Park, S. Y. Choi, and C. Y. Park, "Measurements and analysis of the acoustic signals produced by partial discharges in insulation oil," Current Applied Physics, 2009, 9(2): 296-300.

[32] A. R. Conference, E. Insulation, D. Phenomena, and H. V. Engineering, "Review of partial discharge monitoring techniques used in high voltage equipment," in Annual Report Conference on Electrical Insulation and Dielectric Phenomena 2008, Quebec, Canada, Oct. 26-29, pp. 400-403, 2008.

[33] C. Macià-Sanahuja, H. Lamela, and J. A. García-Souto, "Fiber optic interferometric sensor for acoustic detection of partial discharges," Journal of Optical Technology, 2007, 74(2): 122.

[34] H. Search, C. Journals, A. Contact, M. Iopscience, S. Mater, and I. P. Address, "Extrinsic Fabry-Perot sensor for strain and crack opening displacement measurements from 200 to 900 degrees C," Smart Materials and Structures, 1992, 1(3): 237-242. 